\title{
Role of Calcium Channel Blocking Agents in the Prevention of Atherosclerosis
}

\author{
Bertram Pitt \\ Division of Cardiology, University of Michigan Medical Center, \\ Ann Arbor, Michigan, USA
}

\begin{abstract}
Summary. Calcium channel blocking agents, although effective and widely used in the symptomatic therapy of hypertension and ischemic heart disease, have an uncertain effect on the development of coronary atherosclerosis, plaque rupture, and postrupture thrombosis. Both nifedipine and nicardipine have been shown to prevent the development of new coronary lesions but not the progression of existing lesions in prospective randomized angiographic studies. Verapamil, in contrast, failed to prevent the development of new coronary lesions and had no significant effect on the progression of existing lesions. Diltiazem, although not studied in patients with coronary atheroscleroses, has been shown to prevent the development of post-transplant coronary vascular disease. Despite the beneficial effects of nifedipine and nicardipine on new coronary lesion development, they have not been shown to reduce the incidence of recurrent ischemic events or mortality in the prospective randomized studies that demonstrated their effect on new coronary lesion development. A relatively new dihydropyridine calcium channel blocking agent, amlodipine, is hypothesized to prevent atherosclerosis due to its calcium channel blocking properties as well as by mechanisms independent of its calcium channel blocking properties. This agent has been selected for evaluation in the Prospective Randomized Evaluation of the Vascular Effects of Norvase Trial (PREVENT) to explore whether the use of amlodipine over 3 years will reduce the incidence of early atherosclerotic lesions and, possibly, the progression of existing lesions in both the coronary and carotid arterial beds. Amlodipine could play an important future role in the secondary prevention of ischemic heart disease, but further study and a demonstration of a beneficial effect on recurrent ischemic events is required before any final conclusions concerning its effectiveness are reached.
\end{abstract}

Cardiovasc Drugs Ther 1995;9:21-24

Key Words. calcium channel blockers, coronary atherosclerosis, carotid artery atherosclerosis, nifedipine, nicardipine, verapamil, diltiazem, amlodipine

Calcium channel blockers have found wide application in the therapy of patients with hypertension and ischemic heart disease. They are effective in lowering blood pressure, in relieving exertional angina pectoris and coronary artery spasm, and in improving survival in patients after infarction without left ventricular dysfunction or heart failure. Their role in preventing the development of atherosclerosis is less certain but critical to our understanding of their place in the therapy of both hypertension and ischemic heart disease. If, as suggested by the preclinical data reviewed elsewhere in this supplement, calcium channel blockers prevent the development and possibly the progression of atherosclerosis, they would have a primary role in treating patients with hypertension and ischemic heart disease. If, on the other hand, calcium channel blockers prove not to have a beneficial effect on the development of atherosclerosis, plaque rupture, or thrombosis after plaque rupture, their role will be limited to the symptomatic relief of hypertension and angina pectoris.

\section{Clinical Studies}

\section{Nifedipine}

Several studies have evaluated the effects of calcium channel blockers on the development and progression of atherosclerosis, but their results do not allow a clear conclusion as to their effectiveness. The study by Loaldi et al. [1] was the first angiographic trial to assess the effects of a calcium channel blocker in humans. This study compared the calcium channel blocker nifedipine with the beta-adrenergic receptor blocking agent propranolol and with the nitrate isosorbide dinitrate. Serial angiographic studies suggested that patients who received nifedipine had less progression of their atherosclerosis than those assigned to either propranolol or isosorbide dinitrate. Although relatively small, this study lent emphasis to data from numerous preclinical studies that had shown calcium plays a fundamental role in the development of atherosclerosis. Its conclusions prompted further clinical investigation into the role of calcium channel blockers in preventing atherosclerosis.

Additional support for the potential role of calcium

Address for correspondence: Dr. Bertram Pitt, Department of Pharmacology, M7423 Medical Science Building, The University of Michigan Medical School, Ann Arbor, MI 48109, USA.

Received 16 May 1994, accepted in revised form 7 June 1994 
channel blockers in preventing the development and/ or progression of atherosclerosis came from the study by Gottlieb et al. [2], who investigated the role of nifedipine (compared with placebo) in patients undergoing coronary artery bypass graft surgery. Their prospective randomized study showed that patients randomized to the nifedipine group had significantly more coronary artery bypass grafts that were free of atherosclerosis on serial angiographic follow-up than did those randomized to placebo.

These encouraging studies were followed by the International Nifedipine Trial on Antiatherosclerotic Therapy (INTACT) [3], in which 425 patients with angiographically proven coronary atherosclerosis were randomized to receive either nifedipine or placebo. Patients were followed with repeat quantitative arteriography over a period of 3 years. Patients randomized to nifedipine had a significant reduction in the development of new atherosclerotic lesions [3]. The progression of existing atherosclerotic lesions, however, was not improved.

\section{Nicardipine}

A similar finding (i.e., the prevention of new atherosclerotic lesion development) was shown in the Montreal Heart Institute Study (MHIS) by Waters et al. [4], who randomized 383 patients to nicardipine or placebo and followed them with repeat quantitative arteriography over 2 years. A significant reduction in the incidence of new atherosclerotic lesion formation was noted, but, as shown previously by Lichtlen et al. in the INTACT study [3], no significant effect was evident on the progression of existing atherosclerotic lesions. Data from several studies thus show beneficial effects of calcium channel blockers on the development of coronary atherosclerosis. Once the lesion is established, however, calcium channel blockers appear to have relatively little effect on disease progression.

\section{Verapamil}

In contrast to the other calcium channel blockers, verapamil failed to prevent the development of new coronary lesions and had no significant effect on the progression of existing lesions.

\section{Diltiazem}

Further data on the effects of calcium channel blockers on vascular disease comes from a study by Schroeder et al. [6]; they showed that cardiac transplant patients randomized to receive the calcium channel blocker diltiazem had significantly less obstructive vascular disease than those randomized to placebo. Although the pathology of post-transplant vascular disease is somewhat different from that of atherosclerosis, findings obtained in this study have important implications both for the therapy of the posttransplant patient as well as the understanding of the role of calcium blockers in the development of obstructive vascular disease.

\section{Recurrent Ischemic Events}

Although the data on the effectiveness of calcium channel blockers in preventing the development of coronary atherosclerosis are encouraging, it is somewhat disappointing that in both the INTACT study [3] (with nifedipine) and the study by Waters et al. [4] (with nicardipine) the incidence of recurrent ischemic events was not significantly reduced and in fact trended in the wrong direction: There was a trend towards a higher incidence of recurrent myocardial infarction and death despite the reduction in the development of new atherosclerotic lesions. Although the statistical power of these studies was inadequate to determine the effect of calcium channel blockers on mortality and the follow-up was relatively short, the hope was that agents that were effective in preventing atherosclerosis would reduce recurrent ischemic events, as has been seen with lipid-lowering agents [7].

The failure of the dihydropyridine calcium channel blockers to reduce ischemic events has been noted in meta-analyses by Held et al. [8] and, more recently, by Messerli et al. [9]. These authors note that there is in fact an excess in reinfarction and mortality in patients who receive first-generation dihydropyridine calcium channel blockers. In the meta-analysis by Messerli et al. [9], the odds ratio of reinfarction on a dihydropyridine calcium channel blocker was 1.19 (confidence limits, 0.92-1.53), and there was an odds ratio of 1.16 (confidence limits, $0.99-1.35$ ) for mortality.

The authors note that calcium channel blockers that limit heart rate (e.g., verapamil and diltiazem), in contrast to the dihydropyridine calcium channel blockers, had a beneficial effect on reinfarction and mortality, with an odds ratio of 0.79 (confidence limits, $0.67-0.94$ ) and an odds ratio of 0.95 (confidence limits, 0.82-1.09) for reinfarction and mortality, respectively. They counsel against the use of the dihydropyridine calcium channel blockers and recommend that only heart-rate-limiting calcium channel blockers be used in the postinfarction patient who does not tolerate a beta-blocking agent, because they speculate that heart-rate-limiting agents may have less benefit if heart rate is already limited by a beta-adrenergicblocking agent.

Takenaka et al. [10] also noted an increased incidence of recurrent infarction in 1523 patients randomized to receive nifedipine, diltiazem, or other calcium channel blockers compared with 1029 patients who did not receive a calcium channel blocker (all patients were followed a mean of 13 months). They noted a $4.3 \%$ incidence of reinfarction in those receiving a calcium channel blocker, compared with $2.7 \%$ in those 
who did not $(p<.5)$. The incidence of reinfarction for patients taking nifedipine was $4.7 \%$, which was significantly different from the $2 \%$ incidence in nontreated patients $(p<.05)$, while the difference in patients on diltiazem and the other agents, although increased when compared with the nontreated group, was not statistically significant. The authors concluded that calcium channel blockers increase reinfarction and should not be used as routine therapy.

The reasons for the apparent failure of the firstgeneration calcium channel blockers to reduce recurrent myocardial infarction and mortality despite their proven effects in preventing the development of new atherosclerotic lesions are unclear. These agents have a negative inotropic effect and tend to cause catecholamine release; it is postulated that catecholamine release might predispose towards plaque rupture and sudden cardiac death.

\section{Amlodipine}

We have been interested in a relatively new dihydropyridine calcium channel blocker, amlodipine. Amlodipine binds both to dihydropyridine and nondihydropyridine receptor channels [11]. It has a longer duration of action than the first-generation calcium channel blockers [11]. It is relatively hydrophobic but because of its positive charge it has been suggested to have unique effects on lipid membranes, partially independent of its calcium channel blocking properties [12]. In a primate model, Kramsch et al. [13] have shown that monkeys fed an atherosclerotic diet plus amlodipine had significantly less anatomic evidence of atherosclerosis than animals fed the diet alone, despite comparable increases in serum cholesterol levels and low-density lipoprotein (LDL) cholesterol levels. They also noted that amlodipine significantly decreased the levels of circulating oxidized LDL, insulin, and triglycerides.

They suggest that amlodipine is effective in preventing atherosclerosis by its effect in blocking calcium movement into cells as well as preventing LDL oxidation, and that amlodipine appears to inhibit proliferation of intimal smooth muscle cells and foam cells as well as preventing increases in circulating insulin and triglycerides. Amlodipine also has relatively little negative inotropic effect compared with the firstgeneration calcium channel blockers. In a study by Kloner et al. [14] in a model of coronary artery occlusion and reperfusion, it was found that animals treated with amlodipine did not have any evidence of a negative inotropic effect, evidenced by the absence of an effect of amlodipine on the ratio of change in ventricular pressure to change in time $(\mathrm{dp} / \mathrm{dt})$. Further support for the absence of a negative inotropic effect of amlodipine can be seen in the clinical studies by Packer et al. [15] of patients with heart failure, which documented a beneficial effect of amlodipine on exercise performance in patients with systolic left ventricular dysfunction and heart failure. In contrast to some of the first-generation calcium channel blockers, amlodipine did not cause a release of catecholamines.

\section{Amlodipine in prevention of coronary artery atherosclerosis}

Due to the favorable profile of amlodipine (including an absence of a negative inotropic effect, failure to cause a release of catecholamines, long half-life, positive charge with an effect on the lipid bilayer, antioxidant effect, lack of adverse effects on serum lipids, and excellent patient tolerability) we have begun a study - the Prospective Randomized Evaluation of the Vascular Effects of Norvasc Trial (PREVENT) - to determine the effects of amlodipine on coronary artery atherosclerosis. The details and design of this study will be presented elsewhere, but in brief our primary hypothesis is that amlodipine will reduce the incidence of early atherosclerotic lesions (i.e., those with a 5-20\% diameter stenosis as measured by quantitative coronary arteriography) and, possibly, the progression of existing lesions in patients evaluated by coronary arteriography for clinical reasons. (This effect may occur independent of amlodipine's action as a calcium channel blocker.) The primary endpoint of this study is a change in the average minimal diameter of the lesions after a 3-year follow-up and a decrease in the extent of carotid atherosclerosis (as assessed by B-mode ultrasonography). A secondary hypothesis is that amlodipine will reduce the rate of progression of existing coronary artery lesions and recurrent ischemic events.

The simultaneous evaluation of both coronary and carotid arterial beds should provide unique information both as to the effectiveness of amlodipine as well as to the relationship between the progression of coronary and carotid atherosclerosis. Several current studies are evaluating the effect of various therapeutic agents on carotid atherosclerosis via B-mode ultrasonography, with the assumption that a strategy that prevents carotid atherosclerosis will also prevent coronary atherosclerosis. Although this may be a reasonable assumption with interventions that affect serum lipids, it is less certain in regard to a hemodynamically active agent (e.g., a calcium channel blocker), which, because it lowers blood pressure, may affect different vascular beds differently or at different time courses.

Although this study is not statistically powered to detect the effect of amlodipine on total mortality or the incidence of myocardial infarction, data from 700 patients followed over 3 years should provide important information on the combined incidence of mortality, current myocardial infarction, stroke, and hospitalization for unstable angina pectoris and heart failure. Data will also be analyzed to determine the effectiveness of amlodipine in preventing procedures such as percutaneous transluminal coronary angio- 
plasty, coronary artery bypass graft surgery, and carotid endarterectomy.

\section{Future Directions}

Should the PREVENT trial demonstrate a significant effect on the development and/or progression of carotid and coronary atherosclerosis, amlodipine could play an important role in the secondary prevention of ischemic heart disease. It will, however, be essential to demonstrate that the unique properties of amlodipine translate into a significant reduction in recurrent ischemic events and mortality in view of data suggesting that the first-generation dihydropyridine calcium channel blockers may have an adverse effect on recurrent infarction and mortality [9].

\section{References}

1. Loaldi A, Polese A, Montorsi P, et al. Comparison of nifedipine, propranolol and isosorbide dinitrate on angiographic progression and regression of coronary arterial narrowing in angina pectoris. Am J Cardiol 1989;64:433-439.

2. Gottlieb SO, Brinker JA, Mellits ED, et al. Effect of nifedipine on the development of coronary bypass graft stenoses in high-risk patients: A randomized, double-blind, placebocontrolled trial (abstract). Cireulation 1989;80:II228.

3. Lichtlen PR, Hugenholtz PG, Rafflenbeul W, et al., on behalf of the INTACT group investigators. Retardation of angiographic progression of coronary artery disease by nifedipine. Lancet 1990;335:1109-1113.

4. Waters D, Lesperance J, Francetich M, et al. A controlled clinical trial to assess the effect of a calcium channel blocker on the progression of coronary atherosclerosis. Circulation 1990;82:1940-1953.
5. Schneider W, Roebuck P, Cieslinski G, et al. Beeinflussung der Koronaraskerlose des Menschen durch Kalziumantagonisten? Z Kardiol 1991;80(Suppl 9):63-71.

6. Schroeder JS, Gao SZ, Alderman EL, et al. A preliminary study of diltiazem in the prevention of coronary artery disease in heart-transplant recipients. $N$ Engl J Med 1993;328: $164-170$.

7. LaRose JC. Cholesterol lowering, low cholesterol, and mortality. Am J Cardiol 1993;72:776-786.

8. Held PH, Yusef S, Furberg CD. Calcium channel blockers in acute myocardial infarction and unstable angina: An overview. Br Med $J$ 1989;299:1187-1192.

9. Messerli FH, Weiner DA. Are all calcium antagonists equally effective for reducing reinfarction rate? $A m J$ Cardiol 1993;72:818-820.

10. Takenaka T, Ishikawa K, Kanamasa K, et al. Ca antagonists increased recurrent myocardial infarction (abstract). Circulation 1993;88:I-297.

11. Abernethy DR. The pharmacokinetic profile of amlodipine. Am Heart $J$ 1989;118:1100-1103.

12. Mason RP, Campbell S, Wang S. A comparison of bilayer location and binding for the charged 1,4-dihydropyridine calcium channel antagonist amlodipine with uncharged drugs of this class in cardiac and model membranes. Mol Pharmacol 1989;36:634-640.

13. Kramsch DM, Sharma RC, Hodis HN. Amlodipine suppresses in vivo low-density lipoprotein oxidation, hyperinsulinemia and atherosclerosis in primates (abstract). Circulation 1993;88:I-562.

14. Kloner RA, Hale SL, Alker KJ. Absence of hemodynamic deterioration in the presence of amlodipine following experimental myocardial infarction (abstract). $J$ Cardiovasc Pharmacol 1992;20:837.

15. Packer M, Nicod P, Khandheria BR, et al. Randomized, multicenter, double-blind, placebo-controlled evaluation of amlodipine in patients with mild-to-moderate heart failure (abstract). $J$ Am Coll Cardiol 1991;17:274a. 